\title{
Passive WiFi Radar for Human Sensing Using A Stand-Alone Access Point
}

\author{
Wenda $\mathrm{Li}^{*}$, Robert. J. Piechocki ${ }^{\dagger}$, Karl Woodbridge ${ }^{\ddagger}$, Chong Tang*, Kevin Chetty* \\ ${ }^{*}$ Department of Security and Crime Science, University College London, UK ${ }^{\dagger}$ Department of Electrical and \\ Electronic Engineering, University of Bristol, UK ${ }^{\ddagger}$ Department of Electronic and Electrical Engineering, \\ University College London, UK \{wenda.li, k.woodbridge, chong.tang.18, k.chetty\}@ucl.ac.uk, \\ r.j.piechocki@bristol.ac.uk,
}

\begin{abstract}
Human sensing using WiFi signal transmissions is attracting significant attention for future applications in ehealthcare, security and the Internet of Things (IoT). The majority of WiFi sensing systems are based around processing of Channel State Information (CSI) data which originates from commodity WiFi Access Points (AP) that have been primed to transmit high data-rate signals with high repetition frequencies. However, in reality, WiFi APs do not transmit in such a continuous uninterrupted fashion, especially when there are no users on the communication network. To this end, we have developed a passive WiFi radar system for human sensing which exploits WiFi signals irrespective of whether the WiFi AP is transmitting continuous high data-rate OFDM signals, or periodic WiFi beacon signals whilst in an idle status (no users on the WiFi network). In a data transmission phase, we employ the standard cross ambiguity function (CAF) processing to extract Doppler information relating to the target, whilst a modified version is used for lower data-rate signals. In addition, we investigate the utility of an external device that has been developed to stimulate idle WiFi APs to transmit usable signals without requiring any type of user authentication on the $\mathrm{WiFi}$ network. In the paper we present experimental data which verifies our proposed methods for using any type of signal transmission from a stand-alone WiFi device, and demonstrate the capability for human activity sensing.
\end{abstract}

Index Terms-WiFi Sensing, Doppler Radar, Stand-Alone WiFi Device

\section{INTRODUCTION}

Over the last decade, the increase in the ageing population has led to a focus on addressing the risks which the elderly are exposed to; from falling down and sudden heart attacks, to longer-term conditions relating to mental health, diabetes and cardiovascular disease [1]. The financial and human resource impacts from these emergency events and medical conditions on healthcare services are significant and need to be addressed using a range of approaches. Daily activity monitoring and behavior recognition in residential and working environments are therefore important for both long-term and short-time tasks, and are extremely useful for preventing chronic diseases and health risks, in which early diagnoses are critical. Compared to the traditional monitoring systems like wearables [2] and camera systems [3], WiFi-based sensing technology is considered as an emerging potential solution for various healthcare applications. The reason is, in part, due to the unobtrusive characteristics of RF based sensing and ubiquitous nature of WiFi in both residential and commercial environments which can lead to large coverage areas and flexible deployments. Additionally, unlike camera system, wireless signals are not able to generate images of people, which alleviates many privacy concerns.

The fundamental concept around $\mathrm{WiFi}$ sensing is that when a person moves, the motion of their body will affect the communication channel in terms of signal attenuation, frequency shift and propagation paths. As a result, the timevarying communication channel is related to the physical activities which can be used for various purposes like activity recognition and breathing detection. Early approaches use Received Signal Strength (RSS) from WiFi signal [4], however such systems require intensive offline training to assess the surrounding environment and also suffer from coarse resolution. More recently, WiFi-based human sensing systems have been used to measure the WiFi signal variations in the CSI data, yet these systems [5], [6] treat CSI information as a black box. They use machine learning methods to discover the CSI patterns related to certain activities or position, whereas the lack of quantitative models that connect the CSI information to human activities limits the understanding on WiFi signal dynamic and further development of WiFi sensing technology. Furthermore, it is hard to separate useful signal dynamics from the random noise, and most CSI activity recognition systems are constrained to trail-and-error methods when optimizing the system performance [7]. Conversely, passive radar directly outputs a Doppler spectrogram which can be visually analysed to interpret some basic human motions and can be easily understood for continuous development. Moreover, previous CSI [5], [8], [9] and passive radar systems [10], [11] have used bespoke WiFi devices or high gain antennas with a tapped off reference channel, whereas we use a stand-alone WiFi AP as the illuminator without any modified hardware, swapped antennas or bespoke software.

The term 'stand-alone' refers to a WiFi AP without any specific modification such as an integrated Network Interference Card (NIC), swapped-in high gain antenna for broadcasting, or overwriting the AP's firmware to setup a high transmission data rate. According to the work in [12], the default beacon interval in commodity WiFi APs is 100 Time Units (1TU $=1024 \mathrm{~ns}$ ). Some recent work such as [13] and [14] have focused on using passive radar to exploit the WiFi beacon signal but these studies have all increased the repetition rate of the WiFi beacon bursts from the $100 \mathrm{~ms}$ default setting to 20 
ms (the maximum allowable periodicity on many WiFi APs). These adjustments to make the system usable would again require a level of cooperation with the $\mathrm{WiFi} \mathrm{AP}$ (in this case password authentication) and therefore cannot be relied on for many real-world deployments. Our previous work shows the feasibility of using continuous WiFi signal for breathing detection [15] and activity recognition [16] with passive radar configuration and Line-of-Sight (LoS) environment. This paper further increases the potential of passive WiFi radar to be able work with sparse beacon frame under AP's idle status and Through-The-Wall (TTW) scenario.

To cope the with the low duty cycles associated with beacon frame transmissions, a modified Cross Ambiguity Function (CAF) is implemented to reduce redundant samples and maximize the use of beacon frame. However, this method only allows a limited Doppler detection due to low amount of effective data. To further improve the detection, an external device has been used to simulate a client device for higher data transmission by talking to the WiFi AP continuously. This device uses the probe request-response protocol in $\mathrm{WiFi}$ standard which exchanges information between the WiFi AP and client devices. The probe protocol can be used for requesting the $\mathrm{WiFi} \mathrm{AP}$ information to increase the bandwidth. Our processing also includes a CLEAN algorithm [17] to remove the strong Doppler peak due to strong direct signal and a Constant False Alarm Rate (CFAR) for target detection.

Compared to previous work [5], [8]-[11], the following contributions are made by this study:

- Stand-Alone WiFi AP: Prior to our knowledge, this is the first work study on stand-alone WiFi AP which has no specific modification to either the hardware and software. With the stand-alone system, we further extend the usability of WiFi based sensing for a wider range of use cases.

- Deal with beacon frame: We overcome the limitation of low effective WiFi signal during idle status. Two solutions are provided: modified CAF for sparse beacon frame and a client 'handshaker' to increase the bandwidth for enhancing performance.

- Radio Architecture \& Proof-of-Concept System: The proposed concepts are built into a Software-DefinedRadio (SDR) based Proof-of-Concept (PoC) system. The performance of PoC system is supported by the experiment under TTW scenario with promising results for WiFi sensing.

The rest of this paper is organized as follows: Section II outlines related work in WiFi sensing; Section III describes the basic mechanism of WiFi signal in real-world deployments; Section IV presents the signal processing employed in the standalone WiFi radar system; which is then described in Section V; Finally, experimental results are presented in Section VI and conclusions are given in Section VII.

\section{RELATED WORK}

Generally, there are two major types of WiFi-based activity capture system, which known as the CSI system (collects channel information) and passive radar system (collects Doppler information).

\section{A. CSI System}

A time-series of CSI measurements captures the changes of wireless signal travel through surrounding objects and humans in terms of time, frequency and spatial domain which can be further used for various purposes. Also, different sensing applications have specific requirements of their signal processing techniques and classification/estimation algorithms. All discussed works in this section are based on the Intel 5300 WiFi NIC.

CSI has been widely used in device-free activity recognition. Work [7] implements an activity monitoring system with a package fame rate of $1,500 \mathrm{pk} / \mathrm{s}$. The system contains three receiving channels to monitor at different angel to reduce the limitation of single channel. They also propose a CSI-activity model based on the frequency components of CSI to quantify the relationship between CSI variation and human movement speed to eliminate the effect of environment.

Another work [8] sets the frames rate of $2,500 \mathrm{pk} / \mathrm{s}$ and collects 2,500 CSI values for each of the 180 (2 transmitter antenna $\times 3$ receiver antenna $\times 30$ OFDM subcarriers) CSI streams in one second. This work uses Short-Time Fourier Transform (STFT) technique to transfer the CSI measurements into spectrogram with covered range up to $14 \mathrm{~m}$. However, data can only be collected on a predefined path in a predefined walking direction, which means a training is required for every new environment of activity.

Fall detection is another interesting area in WiFi sensing. Work [18] demonstrates a system that is able to detect falls automatically from other different activities with a package frame rate of $100 \mathrm{pk} / \mathrm{s}$. It exploits the phase and amplitude changes in CSI, and calculates the power profile in timefrequency domain. But the detection performance is affected by the surrounding environment and location due to the direct signal path.

CSI has also been used for fine-grained finger gesture recognition. Work [19] detects the finger gesture with a much lower package frame rate of $20 \mathrm{pk} / \mathrm{s}$ that is more realistic as a real application. It calculates principal components of CSI as the feature vector and classifies finger gesture with Dynamic Time Warping (DTW). This system has a fixed coverage area where the transmitter and receiver are configured as bistatic setup and the target personnel was sitting between them.

Breathing detection by CSI has been shown in [9] with a package frame rate of $20 \mathrm{pk} / \mathrm{s}$. This work quantifies the user location and body orientation based on Fresnel model with an ellipse-shape. It has a similar bistatic setup as [19], whereas the monitoring area is among the middle area between transmitter and receiver.

These works use bespoke $\mathrm{WiFi}$ device to collect CSI information, and manually setup a high package frame rate to ensure the bandwidth, which conflicts with the original communication purpose for a WiFi AP. Also, CSI systems are constrained by the changing of surrounding environment which require various degree of training process as well as the transmitter-receiver location towards monitoring target. 


\section{B. Passive WiFi Radar System}

In addition to CSI systems, passive radar systems have also been used for WiFi sensing. The fundamental aim of passive radar is to use the wireless signal from third-party opportunistic transmitters, and it measures the time difference between the signal arriving directly at the transmitter and the signal arriving via reflection from the object of interest. Different from CSI systems, a typical passive radar contains two coherent channels: a surveillance channel which points to the surveillance area and a reference channel which points to the WiFi AP to reconstruct transmitted signal. Passive radar uses the Cross Ambiguity Function (CAF) to calculate range (bistatic distance) and Doppler (bistatic velocity) information. However, due to the bandwidth of WiFi signal is limited (20$40 \mathrm{MHz}$ ), normally only the Doppler information has been used for indoor scenario.

An early attempts of passive WiFi radar is shown in [17] which demonstrates the feasibility of using WiFi signal to detect personnel at a stand-off $(12 \mathrm{~m})$ distance and TTW scenario. It successfully detects the Doppler of a moving personnel and improves the Signal-to-Interference Ratio (SIR) with a CLEAN algorithm. Its reference channel was been connected directly to the WiFi AP for a perfect signal reconstruction which eliminates the risk of interference.

Work [10] further verifies the passive WiFi radar in an outdoor scenario, and able to correct both range and Doppler information for a moving car and a running human. This work connects a high gain $(30 \mathrm{~dB})$, narrow beam $\left(15^{\circ}\right)$ antenna to the WiFi AP to improve the SIR, and puts both transmitter and receiver antenna at same location. This configuration is more like a Frequency-Modulated Continuous-Wave FMCW radar but transmits WiFi signal.

Lately, work [20] built a prototype base on the SoftwareDefined-Radio (SDR) platform with low-complexity pipeline design to enable real-time processing ability. This system shows some preliminary results for several applications like TTW activity recognition and finger gesture recognition within laptop area. It has a classical passive radar configuration but assumes that reference channel is stable without any interference from surrounding, and also modified a continues WiFi signal.

Passive WiFi radar has also been used for vital sign detection, in [15] we demonstrate that the micro-Doppler can be obtained from chest motion as a representative of breathing signal. With appropriate signal processing, it can be further applied to multiple tasks [21]. However, due to the complicated geometry, both [15] and [21] systems are affected by the location among transmitter, receivers and personnel.

All above works have made some changes to the WiFi AP to enable a high bandwidth signal to broadcast or attached a high gain antenna to the AP. Such configurations are hard to direct applying to real-world scenarios in terms of the bandwidth in communication network and high cost for antenna. In addition, these system geometry are too optimal to be real, like the perfect reference channel and active radar like geometry.
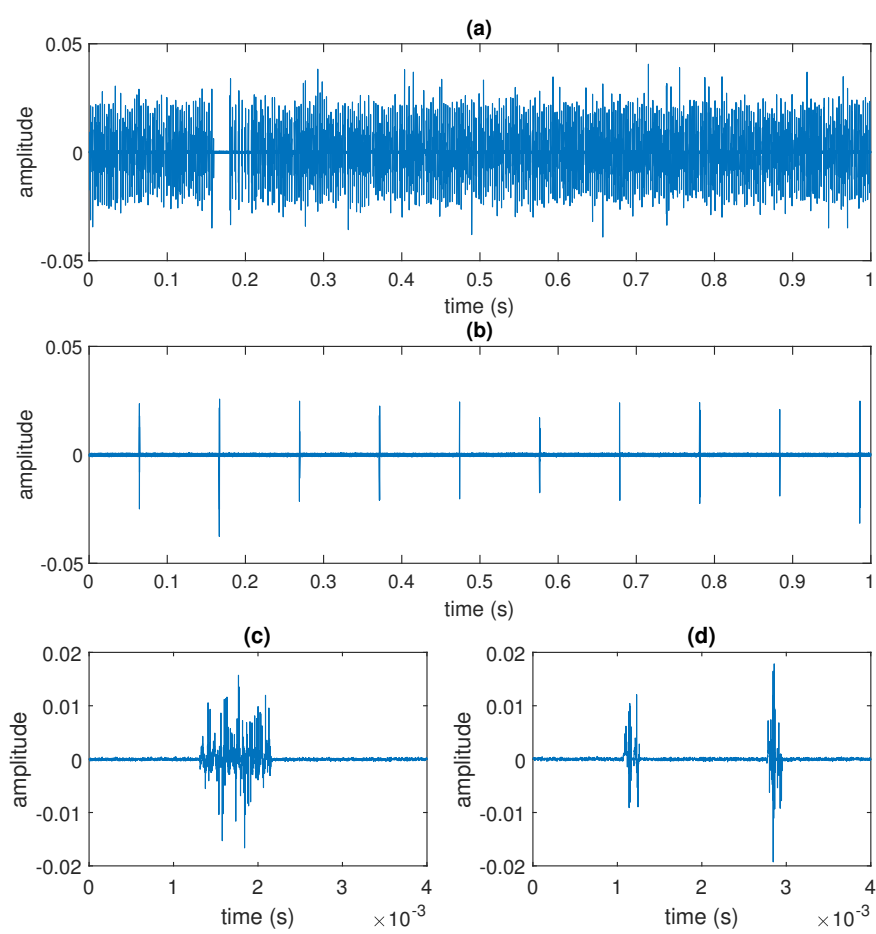

Fig. 1: An example of WiFi status (a) transmission status (full bandwidth WiFi signal), (b) idle status (only contains beacon frame), (c) zoom in of beacon frame and (d) zoom in of probe request \& response frame.

\section{WiFI PROTOCOLS FOR SENSING}

\section{A. WiFi AP Status}

In passive sensing, the WiFi signal is considered as an independent pseudo-noise waveform that is either OFDM modulated for high date-rate communications or a lowerbandwidth signal generated when the WiFi AP is in an idle state. As discussed in last section, most current WiFi sensing systems [8], [10], [18], [20] rely on the high frame rates to generate sufficient CSI/Doppler information. However, such situation may not always true in case of real life, as communication devices are designed to be bandwidth effective. Here we provide two examples of WiFi signals; first example shows a busy AP which is transmitting data continuously, named as the transmission status; and the second example illustrates the case when there is no internet usage so contains only beacon frames, named as the idle status. The data for both examples were captured for one second, and their time domain representations are illustrated in Fig 1. As shown in the figure, the WiFi signal occupies almost full time domain (>90\%) during the transmission status with small time gap. This provides a large amount of effective data to be used by the passive radar system. In comparison, during idle status, the beacon frame has a small duty cycle with large spaces of redundancy. In fact, manual measurements show that the duty cycle of the beacon frame is $0.42 \%$ of that of a data transmission signal. As a consequence, the effective energy one could reuse for processing from the beacon frame is very limited, and as a result the classical CAF processing struggles 


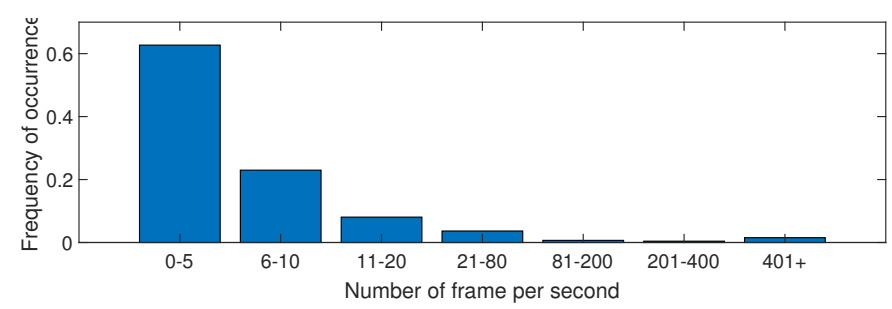

Fig. 2: Frame rate captured over 24 hour period.

to work effectively.

\section{B. Beacon and Probe Response Frame}

Typically, beacon frames are transmitted periodically to declare the presence of a WLAN with information about the network such as its modulation type, code rate, compatible standards etc. A wireless enabled device generally scans all RF channels to search for WiFi beacons, which then allows the device to choose to connect the optimal network. Increasing the beacon interval will reduce the load on the network and increase throughput, however it delays the association and roaming processes. Alternatively, decreasing beacon interval decreases throughput for users and increases the network load. A zoom in of beacon frame is shown in Fig 1(c) with a duration less than $0.5 \mathrm{~ms}$.

The WLAN clients use probe request frames to scan the area for availability of WLAN network. In response to the received probe request, the network sends out a probe response frame when parameters are compatible. Probe response frames are exclusive to physical layer in use as mentioned in beacon frame. A zoom in of probe request and response frame is shown in Fig 1(d). As it can be seen, the first frame with lower amplitude is a probe request signal and the second is probe request frame due to WiFi AP has higher transmission power. In this work, we use a client simulator to trigger probe request and response frames at relatively high transmission rates. This approach is similar to the mechanism in CSI system but avoids any firmware modifications and hardware changes for WiFi AP.

Both the beacon and probe response frames are not encrypted in IEEE 802.11 family of standards. The difference is that the beacon frame broadcasts constantly from a WiFi $\mathrm{AP}$, whereas probe response signal is a response to the client device. Both signals can be used for opportunistic sensing, but their performance depends on the amount of effective data. The duration of beacon and probe response frame are varied depending on the standard, setting and information contained.

\section{Monitoring a public access WiFi Access Point}

To better understand the WiFi usage in a real scenario, we use the WiFi network analysis tool 'Wireshark' to monitor the WiFi traffic from the 'Eduroam' wireless network over a 24 hour period. Eduroam is an university wide WiFi network and the AP of interest was located in an office consisting of an approximately 40 staff and students who are present during typical office hours. The transmitted frame number

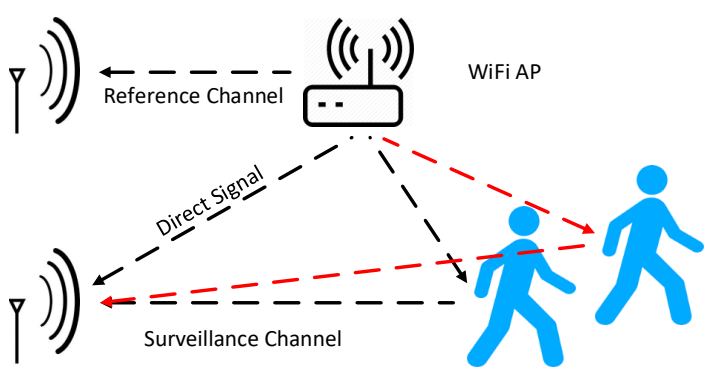

Fig. 3: Passive WiFi radar sensing.

versus frequency of occurrence is shown in Fig 2. It is found that even during the working hours (between $9 \mathrm{am}$ to $5 \mathrm{pm}$ ), the peak is around 100 frames per second which is much lower than the request frame rate in the previous activity recognition work [7], [8], [19]. Outside of working hours, the frame rate drops to around 4-5 frames per second which is of similar order to the beacon frame rate. In addition, it is reasonable to assume WiFi usage in a residential area will be lower than this level due to lower user numbers. Thus, it is necessary to consider the effect of low frame rates when designing a real WiFi sensing system.

\section{Signal Processing for Passive WiFi Radar}

\section{A. Preliminary of Passive WiFi Radar}

Passive radar systems employ two synchronised receiver channels, the surveillance channel records reflected WiFi signals from the monitoring area whilst the reference channel records the signal from the transmitter. In a typical indoor scenario, as illustrated in Fig 3, WiFi transmissions propagate through the space and the signal environment is characterised by both the topology and clutter. If a person is present in the environment, additional changes are introduced to the signal which correspond to the effects of human presence in the environment. If there is no one or no motion in the environment, the signal will be relatively stable. However, as shown by the red lines in Fig 3, along with the person moving to a new location, the geometry of passive radar system is changing which results in the change of amplitude attenuation and phase shift in WiFi signal.

\section{B. Collecting Doppler Information}

In IEEE 802.11 standard, signals are modulated by OFDM scheme [22]. This also applies to the beacon and probe response signal. Let the transmitted OFDM signal defined as:

$$
x(t)=\frac{1}{\sqrt{N}} \sum_{n=0}^{N-1} a_{n} e^{j 2 \pi n t}
$$

where $N$ is the number of OFDM symbols for each carrier $a_{n}$ and $n$ is the index of OFDM symbol. The received signal $y(t)$ consists of both direct signal and target reflections. These reflections from a stationary clutter or a moving person can be described by a summation of delayed and phase shifted transmitted signal. The received signal can be written as: 


$$
y(t)=\sum_{p} A_{p} e^{j 2 \pi f_{d} f_{c} t} x(t-\tau)+n(t)
$$

where $p$ is the number of reflected paths, and $A_{p}, \tau, f_{d}$ are the attenuation factor, delay, Doppler shift for $p$-th path respectively, $\mathrm{n}(\mathrm{t})$ is the Additive White Gaussian Noise (AWGN).

In passive radar, CAF processing is commonly used to obtain the range $\tau$ and Doppler $f_{d}$ information by taking Fast Fourier Transform (FFT) of cross-correlated signals from surveillance channel $S_{\text {sur }}(t)$ and reference channel $S_{r e f}(t)$. In terms of a passive WiFi radar, $S_{\text {sur }}(t)$ represents the $y(t)$ which is collected from the surveillance area and $S_{r e f}(t)$ recreates the transmitted signal $x(t)$ without any interference as possible. In this case, $\mathrm{CAF}$ equation can be written as:

$$
C A F\left(\tau, f_{d}\right)=\int_{0}^{T_{i}} S_{s u r}(t) S_{r e f}^{*}(t-\tau) e^{j 2 \pi f_{d} f_{c} t} d t
$$

where $T_{i}$ is the integration time which defines the Doppler resolution as: $\Delta f_{d}=1 / T_{i}, *$ is the complex conjugate. However, above equation requires a high computational load which prohibits our system for operating in real-time. Thus, a batch processing technique [20] has been used for complexity reduction. This is achieved by dividing a long sequence into several short batches so that the cross-correlation and FFT process over each batch are faster.

One of the limitations of $\mathrm{Eq} 3$ is that it cannot generate meaningful Doppler signature during the idle status (Fig 1(b)) due to the ultra-low-density of the beacon signal, as well as the high amount of redundant noise. To that end, the modified CAF is proposed to maximize the effective data contained within the beacon signal. The idea is to synchronize and extract the beacon signal before them passing to the CAF. This can ensure only useful data are collected and processed, whereas the noisy data can be filtered out. In this work, beacon synchronization is implemented based on the energy curve $X_{i}$ which is calculated as the cumulative sum of the absolute value of WiFi signal. Then the normalized energy curve $X_{i}^{\prime}$ as suggested in [23], is calculated as:

$$
X_{i}^{\prime}=X_{i}-i \delta=\sum_{k=0}^{i}\left(x_{k}^{2}-i \delta\right)
$$

where $k$ is the index of signal, $i$ is loop variable ranging through all samples. $\delta$ is a negative trend and depends on the total energy of the selected signal $X_{N_{e}}$ with window length of $N_{e}$ as determined by $\delta=\frac{X_{N_{e}}}{\alpha \cdot N_{e}}$. In this work, $N_{e}$ is the length of signal of one integration time $T_{i}$.

A demonstration of one second beacon synchronization is shown in Fig 4. The energy curve for beacon signal Fig 4(a) is calculated in Fig 4(b), and the corresponding normalized curve is plot in Fig 4(c). Afterwards, we search the smallest value within each beacon interval as the start time of a beacon frame, for example Fig 4(c) is an example of $100 \mathrm{TU}$. Afterward, the modified CAF with synchronized beacon signal can be written as:

$$
C A F\left(\tau, f_{d}\right)=\sum_{n=0}^{N_{b}-1} \int_{0}^{T_{b}} S_{\text {sur }}^{n}(t) S_{r e f}^{n}{ }^{*}(t-\tau) e^{j 2 \pi f_{d} f_{c} t} d t
$$
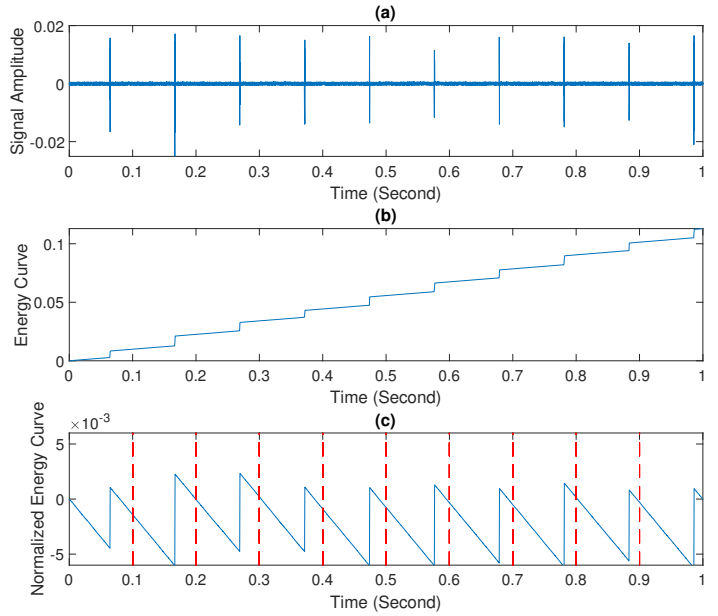

Fig. 4: The beacon synchronization process.
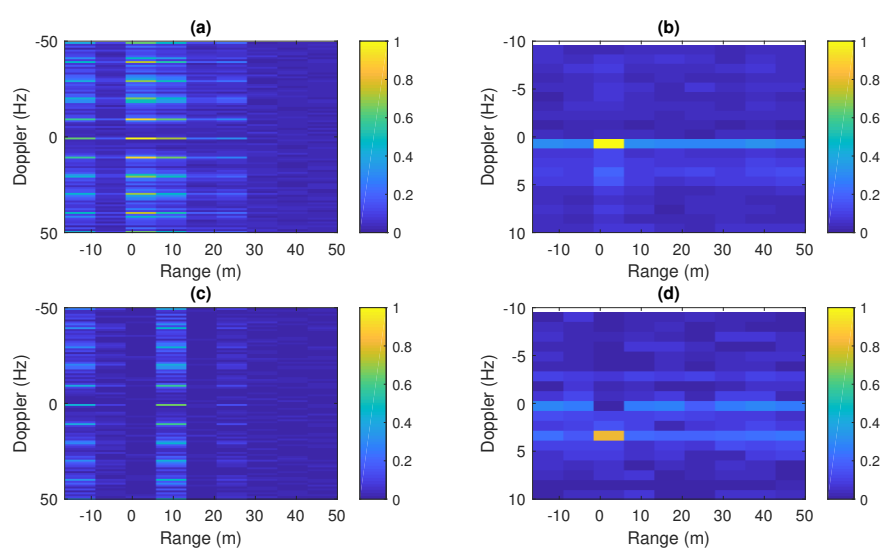

Fig. 5: An example of CAF surface during WiFi idle status, (a) classical CAF (Eq 3), (b) modified CAF (Eq 5), (c) classical CAF with CLEAN algorithm, and (d) modified CAF with CLEAN algorithm.

where, $N_{b}$ is the batch number ans id equivalent to the number of Doppler bins or the number of beacon, $T_{b}$ is the time duration of each batch or the duration of a beacon, and $n$ is the index of batch. To optimise performance, the beacon synchronization is designed based on the signal recorded in the reference channel as it has a higher SNR and is subject to less fluctuations that the signal measured in the surveillance channel. Note however, there are still interference components in reference channel (for example, other data packets or from a WiFi AP transmitting in the same frequency band), which may lead to synchronization distortions.

Examples of a CAF output surface using classical CAF (Eq 3) and modified CAF (Eq 5) after processing the beacon signal are shown in Fig 5(a) and (b). This data is captured from a walking person. As it can be seen, there are significant sidelobes in both Doppler and range on CAF surface with classical $\mathrm{CAF}$, and no meaningful information can be extracted. We attribute this output to the sparse nature of beacon frame. For this reason, the modified CAF is applied. Visual inspection of the output shows a much cleaner surface with a dominant 


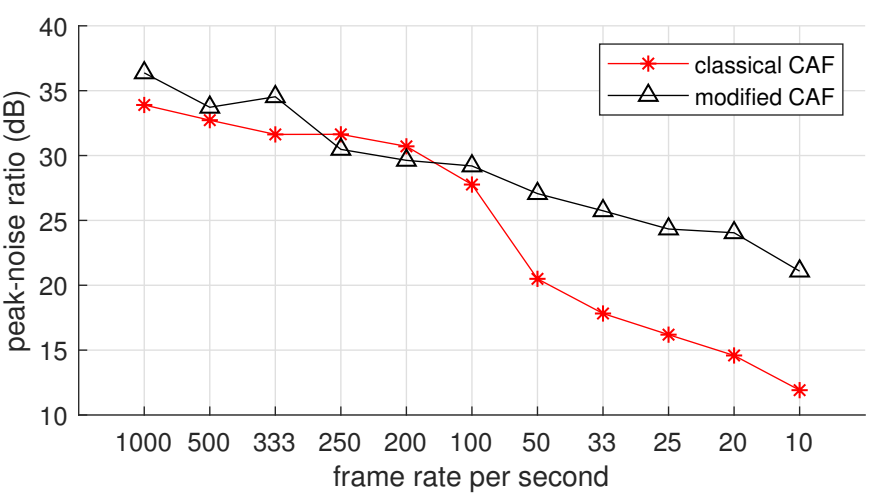

Fig. 6: Peak-Noise Ratio (PNR) vs frame rate.

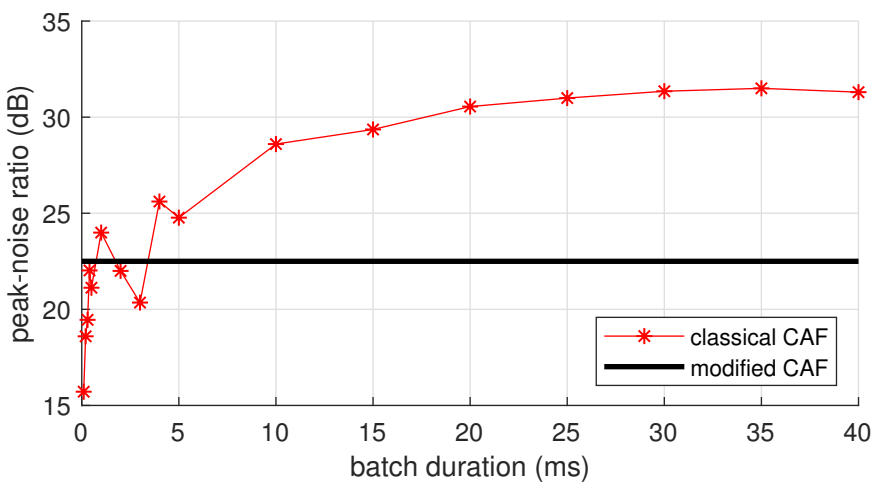

Fig. 7: Peak-Noise Ratio (PNR) vs the batch duration.

peak at zero range/Doppler bin. Note that the boundary of Doppler is limited in sparse mode since there are only ten WiFi beacon bursts available in each second which can be used in the batched processing. This means the maximum detectable boundary is five Doppler bins for 1 second (in both positive and negative domain). To better demonstrate the Doppler pulse, the integration time $T_{i}$ for sparse mode was set as two seconds so that twenty beacons are available in the range-Doppler surface.

To further demonstrate the effect of frame rate in the CAF processing, the Peak-Noise Ratio (PNR) between the dominant Doppler peak and rest CAF surface is measured. Higher PNR generates a higher quality Doppler spectrogram. For modified CAF, the frame rate also determines its batch number $N_{b}$, where the classical CAF has a constant $N_{b}=100$. The PNR plot of both CAF processing are shown in Fig 6. As it can be seen, there is a significant improvement of proposed modified CAF, in average, modified CAF delivers $8 \mathrm{~dB}$ higher PNR than the classical CAF between 10 to 50 frame rate. PNR values become similar after 100 frame rate with very small difference between two CAF processing.

Another important factor is the batch duration $T_{b}$ which defines the length of data used for the cross-correlation processing, and also affects the noise level in CAF surface. Generally, a longer batch duration leads to a lower noise level, but requires more computational power. A plot of PNR versus batch duration is shown in Fig 7. As it can be seen, PNR of the classical CAF improves gradually until $10 \mathrm{~ms}$ then remains constant thereafter. For the modified CAF, PNR is constant because the duration of beacon signal is fixed at $0.8 \mathrm{~ms}$. In this work, batch duration of both enhanced mode and transmission status were set as $10 \mathrm{~ms}$ to fully use the received data, i.e. $T_{i}(1 s)=N_{b}(100) \times T_{b}(10 \mathrm{~ms})$.

\section{Cancellation of the Direct Signal Interference}

The surveillance channel of a passive radar system consists of a direct signal interference (DSI) component, target clutter, Doppler-shifted target echoes, and thermal noise. The expression of received signal in surveillance channel can be written as:

$$
S_{\text {sur }}(t)=S_{d s i}(t)+S_{\text {clutter }}(t)+S_{\text {tar }}(t)+n(t)
$$

where $S_{d s i}(t)$ is the signal from direct path, $S_{\text {clutter }}(t)$ is the signal reflected from surrounding clutter and $S_{t a r}(t)$ is the signal reflected from target. Replacing $S_{s u r}(t)$ in Eq 6 with its full expression from Eq 5 allows the final CAF surface to be written as the sum of the CAF surfaces of each components as:

$$
\begin{aligned}
C A F\left(\tau, f_{d}\right) & =\sum_{l_{l}=1}^{L_{L}} C A F_{d s i}^{\left(l_{l}\right)}\left(\tau, f_{d}\right)+\sum_{l_{m}=1}^{L_{M}} C A F_{\text {clutter }}^{\left(l_{m}\right)}\left(\tau, f_{d}\right) \\
& +\sum_{l_{n}=1}^{L_{N}} C A F_{\text {tar }}^{\left(l_{n}\right)}\left(\tau, f_{d}\right)
\end{aligned}
$$

where $C A F_{d s i}, C A F_{t a r}$ and $C A F_{n}$ are the CAF surface due to DSI, target and noise respectively. $L_{L}$ represents the number of reflected paths due to DSI, $L_{M}$ represents the number of reflected paths due to the surrounding clutter and $L_{N}$ represents the number of reflected paths due to the target. We note that in Eq 7, item $\sum_{l_{l}=1}^{L_{L}} C A F_{d s i}^{\left(l_{l}\right)}\left(\tau, f_{d}\right)$ would be expected to generate a main peak in the zero Doppler bins, whereas item $\sum_{l_{m}=1}^{L_{M}} C A F_{\text {clutter }}^{\left(L_{m}\right)}\left(\tau, f_{d}\right)$ can produce both range and Doppler sidelobe responses.

There are two types of technique to implement the CLEAN algorithm for removing the effect from DSI and stationary clutter, known as pre-processing and post-processing. Preprocessing techniques aim to perform subtractions in the signal domain rather than in the CAF surface, however this process requires significant computational power considering the size of raw WiFi signal received by SDR system. Alternatively, post-processing method subtracts the interference at CAF surface, and is a less complex approach requiring lower computational power. We therefore focus more on postprocessing techniques to achieve real-time outputs.

In this work, we adopt the method suggest by [17] with a shifted magnitude-scaled and phase-corrected version of the self CAF surface to provide a model of the cross-ambiguity surface. Let $C A F^{k}\left(\hat{\tau}, \hat{f}_{d}\right)$ represents the cleaned CAF mapping at $k_{t h}$ iteration, it can be written as:

$$
C A F^{k}\left(\hat{\tau}, \hat{f}_{d}\right)=C A F^{k-1}\left(\tau, f_{d}\right)-\alpha^{k} C A F_{\text {self }}\left(\tau-T_{k}, f_{d}\right)
$$

where $\alpha^{k}$ is the maximum absolute value of $C A F^{k}\left(\tau, f_{d}\right), T_{k}$ is the phase shift factor refers to the $\alpha^{k}$. CAF self is the self CAF over the reference channel. The phase of $C A F^{k}\left(\hat{\tau}, \hat{f}_{d}\right)$ 
is shifted by multiplication with a complex phasor $e^{j \Delta \phi}$, where $\Delta \phi$ is the difference in phase between the peak in $C A F^{k}\left(\hat{\tau}, \hat{f}_{d}\right)$ and the peak found along the zero Doppler bin. From observation, one iteration $(k=1)$ is sufficient for our system.

The cleaned CAF surface of Fig 5(a) and (b) are shown in Fig 5(c) and (d) respectively. For classical CAF, even after the CLEAN algorithm, there is still no meaningful Doppler information. In contrast, the dominant peak in modified CAF surface has been successfully removed. As a result, the desired peak is present clearly on the map at $5 \mathrm{~Hz}$ which corresponds to the persons velocity.

Afterwards, a Doppler spectrogram $D\left(f_{d}, t\right)$ is generated by extracting one column with maximum value in the CAF surface.

\section{Motion Indicator}

In this work, we consider a person as being in one of two states: an active state (e.g. walking, body swing and other types of full/part body movement) and a stationary state such as sitting or standing still, where only the motion of the chest wall during breathing is detectable. Thus it is necessary to distinguish these two periods, since their Doppler signatures are significantly different.

This is based on the fact that human movements generate much higher power in terms of Doppler pulse. When there are no human movements, the power in Doppler spectrogram is mostly low since there is no frequency shift in the surveillance channel. In this work, we consider it as a pulse detection problem by estimating the noise level $\hat{N}(t)$ using a moving average (MA) algorithm during the stationary period as $\hat{N}(t)=\frac{1}{w} \sum_{t=0}^{w}\left|D\left(f_{d}, t\right)\right|^{2}$, where $w$ is the window length which is set as 5 seconds.

We set the detection threshold as four times of the noise level, so that a movement can be detected when the Doppler power deviates from the average noise level. Once a movement is detected, we use a standard constant false-alarm rate (CFAR) algorithm to detect the Doppler response associated with a moving person, and record the spectrogram.

\section{E. Breathing Response}

During stationary periods, the velocity of the chest wall is considerably lower than that of body movements. Detection of the periodic chest wall motion associated with breathing can therefore be achieved with a higher Doppler resolution by extending the integration time $T_{i}$ used in Eq 3 . However, the target's velocity is assumed constant during the period of integration time whereas this is not suitable for our system to capture fast movement. Thus we use a micro Doppler extraction method to extract the breathing signal $B(t)$ while maintaining the Doppler resolution as normal. The idea is to use the macro imbalance between the Doppler positive and negative domain caused the chest motion.

$$
B(t)=\sum_{f_{d}=-\frac{1}{2} M A X\left\{f_{d}\right\}}^{\frac{1}{2} M A X\left\{f_{d}\right\}} D\left(f_{d}, t\right)(n) *\left\lceil\frac{M A X\left\{f_{d}\right\}}{2}-i\right\rceil
$$

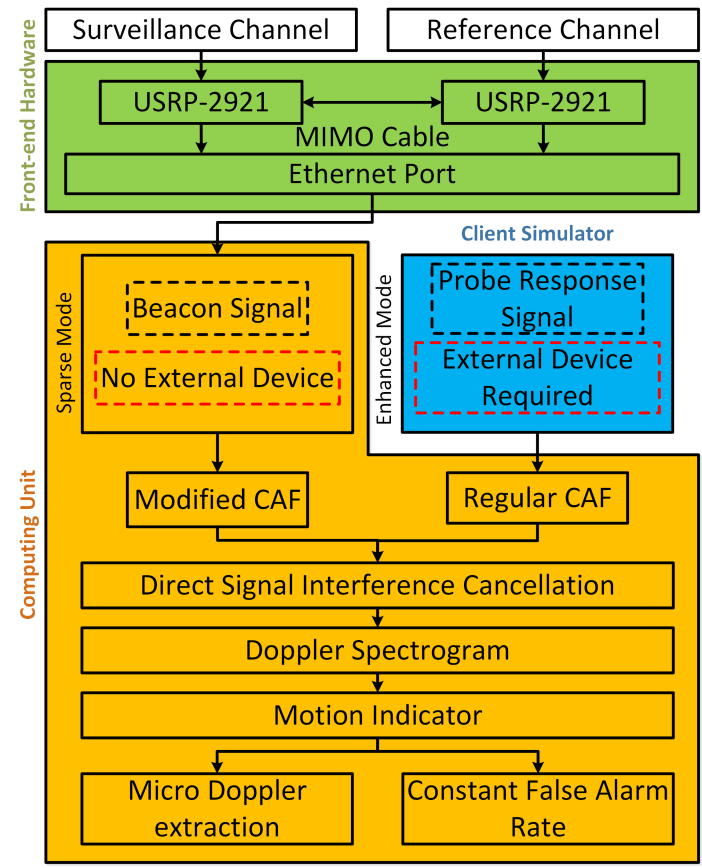

Fig. 8: Block diagram of proposed system.

where $i$ is the index of Doppler bin, $M A X\left\{f_{d}\right\}$ represents the maximum of Doppler bins in Doppler spectrogram $D\left(f_{d}, t\right)$, it is equivalent to the number of beacon in terms of sparse mode and was set as 100 in terms of enhanced mode and during transmission status. When the target is in stationary, the breathing response signal $B(t)$ can be obtained directly from the spectrogram as it only contains chest movement.

\section{F. Target Detection}

A CFAR threshold has been used to detect the target in CAF surface and further reduce the levels of noise and interference. There are multiple interference sources which may arise from: incorrect beacon synchronization that captures an ineffective signal; interference from WiFi signals from other AP; and other packet transmission interrupting the beacon synchronization.

$$
\Lambda=\frac{1}{N_{\tau} \cdot N_{f_{d}}} \sum_{i=1}^{R_{\tau}} \sum_{j=1}^{R_{f_{d}}} C A F\left(\tau_{i}, f_{d_{j}}\right)
$$

where $\Lambda$ is the threshold mapping for CAF. $i$ and $j$ are the index for range and Doppler bin, $N_{\tau}$ and $N_{f_{d}}$ are the training length in range and Doppler bin respectively. This threshold mapping is then used for normalizing the power and remove the noise as $P(i, j)=|C A F(i, j)|^{2} / \Lambda$. For $P(i, j)<1$ are considered as noise and replaced with zeros, others are considered as activities.

The effectiveness of above signal processing will be presented in Section VI.

\section{SySTEM IMPLEMENTATION}

\section{A. System Overview}

The proposed passive WiFi radar system is implemented based on the popular USRP platform. The overall block 
TABLE I: System parameters for each mode.

\begin{tabular}{|l|l|l|l|}
\hline & sparse mode & $\begin{array}{l}\text { enhanced } \\
\text { mode }\end{array}$ & $\begin{array}{l}\text { transmission } \\
\text { status }\end{array}$ \\
\hline WiFi signal & $\begin{array}{l}\text { beacon } \\
\text { frame only } \\
(100 \text { TU, } \\
\text { default } \\
\text { setting) }\end{array}$ & $\begin{array}{l}\text { probe response } \\
\text { frame (13 TU) }\end{array}$ & $\begin{array}{l}\text { data frame } \\
\text { (varied time } \\
\text { gap, base on } \\
\text { ine } \\
\text { usage) }\end{array}$ \\
\hline effective data & around $0.4 \%$ & around 18\% & around 90\% \\
\hline $\begin{array}{l}\text { integration time } \\
T_{i}\end{array}$ & 2 second & 1 second & 1 second \\
\hline $\begin{array}{l}\text { number of batch } \\
N_{b}\end{array}$ & 20 & 100 & 100 \\
\hline batch duration $T_{b}$ & $0.8 \mathrm{~ms}$ & $10 \mathrm{~ms}$ & $10 \mathrm{~ms}$ \\
\hline $\begin{array}{l}\text { maximum } \\
\text { detectable } \\
\text { velocity } \\
\text { max }\left\{f_{d}\right\}\end{array}$ & $\begin{array}{l}6.625 \\
(\text { cannot } \\
\text { increase) }\end{array}$ & $\begin{array}{l}6.25 \mathrm{~m} / \mathrm{s} \text { (can } \\
\text { increase) }\end{array}$ & $\begin{array}{l}6.25 \mathrm{~m} / \mathrm{s} \text { (can } \\
\text { increase) }\end{array}$ \\
\hline bandwidth & $1 \mathrm{M}$ & $1 \mathrm{M}$ & $1 \mathrm{M}$ \\
\hline
\end{tabular}

diagram of our passive WiFi radar is presented in Fig 8. It contains mainly three parts: radio frequency front-end, passive radar signal processing and a client simulator.

The front-end hardware uses two NI USRP-2921 [24] each contains a tunable RF transceiver for acquiring the wireless signal and two ADCs for digitization. A MIMO-cable is used to share the clock source between two USRPs to synchronize the reference and surveillance channel. Surveillance channel is equipped with a $18 \mathrm{~dB}$ Yagi antenna, and reference channel is equipped with a $14 \mathrm{~dB}$ Yagi antenna. The collected raw data is then transmitted to a computing unit, which is a laptop in this work, through an Ethernet port. Note that the NI-USRP is a tool which allows rapid prototyping and testing, a real-world passive WiFi sensing system would require lower technical specification and a less complex radio architecture making it significantly lower cost.

The key feature of our system is the capability of using stand-alone WiFi AP for personnel monitoring. Thus, a modified CAF (using eq 5) and a client simulator are implemented to cope with the sparse beacon signal. The signal processing for passive radar described in Section IV is implemented with LabVIEW. It first calculates the CAF surface from either sparse or enhanced mode based on the quality of the Doppler signature and the density of WiFi signal. Afterwards, a motion indicator identifies the activity level and chooses the desired output. A pipeline structure [15] has been used to divide the long serial signal processing of passive radar signal processing into short parallel processing. This structure allows the system to run in real-time on a laptop.

The client simulator adopts the idea of probe response protocol to generate higher bandwidth with more effective signal. In our system, it is used aligned with the enhanced mode during WiFi idle status. This device can be activated when there is a need of detailed detection during the WiFi idle status. Note that, the regular CAF is also applied to $\mathrm{WiFi}$ transmission status as it has high frame rate.

Table I summarizes the system parameters for our passive WiFi radar system. The maximum detectable velocity can be calculated as: $\max \left\{f_{d}\right\}=N_{b} \times \Delta f_{d} \times f^{\prime} / 2$, where $\Delta f_{d}=$ $\frac{1}{T_{i}}$ is the Doppler resolution and $f^{\prime}=\frac{c}{f_{c}}=\frac{3 * 10^{8}}{2.4 * 10^{9}}$ is the relative velocity corresponding to each $\mathrm{Hz}$ in Doppler which
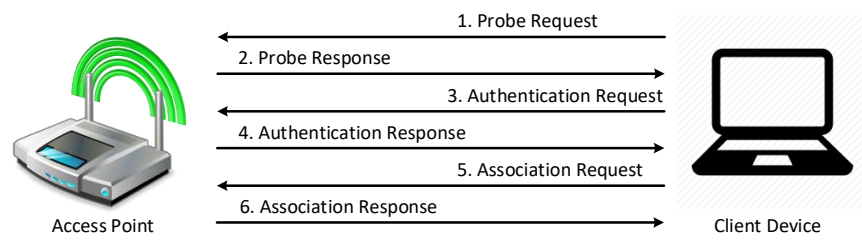

Fig. 9: Life-cycle of probe response signal.

is $0.125 \mathrm{~m} / \mathrm{Hz}$. The factor 2 is used for representing both positive and negative domain. The major limitation of sparse mode is the maximum detectable velocity $(0.625 \mathrm{~m} / \mathrm{s})$ which is due to the number of beacon of frame as 10 frames per second. To better demonstrate the Doppler signature, we use 2 seconds of integration time $T_{i}$ in sparse mode to double the $N_{b}$ but half the $\Delta f_{d}$. Thus the $\max \left\{f_{d}\right\}=N_{b}$ remains the same. In comparison, enhanced mode has no limitation on maximum detectable velocity by having higher $N_{b}$ as in Eq 3.

\section{B. Implementation of Client Simulator}

A Raspberry Pi running Kali Linux has been developed to stimulate a client device which continuously sends probe request frames to the target WiFi AP. The waveform of a response frame is shown in Fig 1(d). It is processed according to the 802.11 protocol [25] and includes three consistent request frames and three response frames as shown in Fig 9. To avoid the mechanism in WiFi protocol that prevents a device repeatedly sending the request frame, the client simulator changes its MAC address after each probe request is sent.

In our tests, the maximum achievable frame rate for current setup is 75 probe requests per second (approximately $13 \mathrm{TU}$ ) which provides much higher density than the default setting of beacon signal (100 TU). A higher frame rate could be achieved with more powerful system like a WiFi NIC. Despite the enhanced mode being able to generate a large amount of effective data to be used by our passive WiFi radar system, the bursty nature of probe response signal means there are still considerable time gaps compared to the WiFi transmission status (Fig 1(a)).

\section{EXPERIMENTAL RESUlts}

The test area consisted of an individual room of dimensions $4.3 \mathrm{~m} \times 4.6 \mathrm{~m}$, separated by a $16 \mathrm{~cm}$ thick wall made of both brick and plasterboard. A stand-alone WiFi AP (Model: DWL-2000AP+) was placed in the corner of the test area whilst the passive WiFi radar system was located outside of the room. The reference and surveillance antennas were directed towards the WiFi AP and center of the room. A large file was transmitted through the WiFi AP for experiments which required high-data rate signal transmissions; termed transmission status. For experiments which required only WiFi beacon signal transmissions; termed the idle status, the WiFi AP was left alone. This respective WiFi signal transmissions are illustrated in Fig 1(a) and (b), and the experimental layout is shown in Fig 11. 


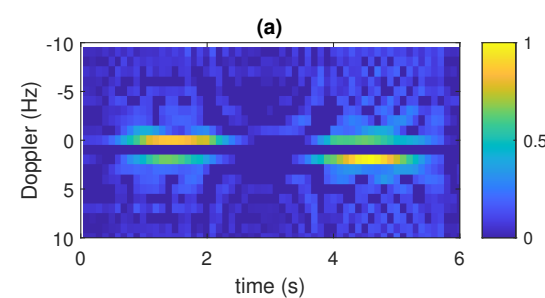

(d)

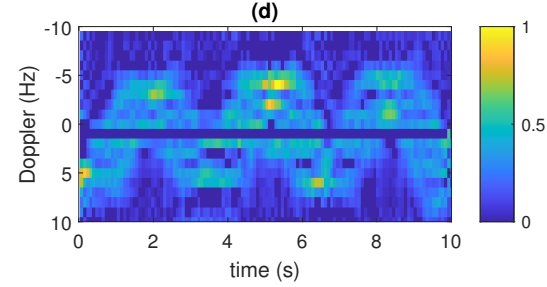

(g)

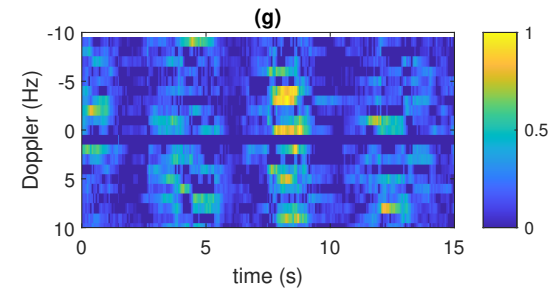

(b)

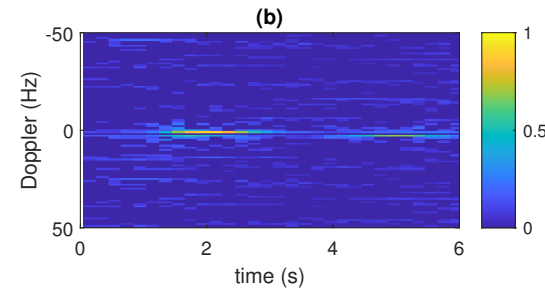

(e)

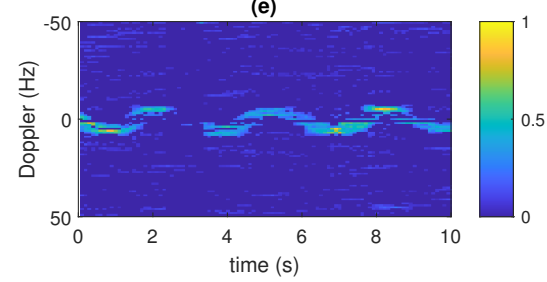

(h)

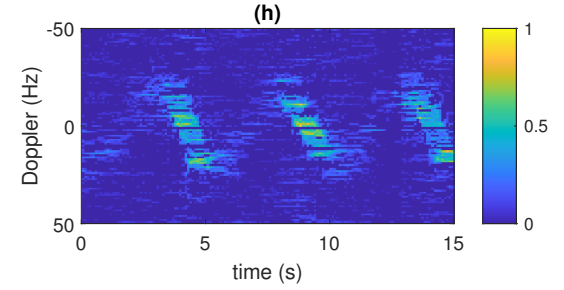

(c)

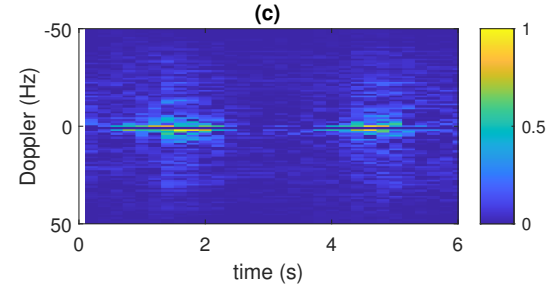

(f)

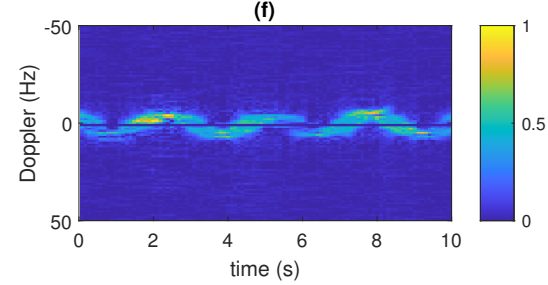

(i)

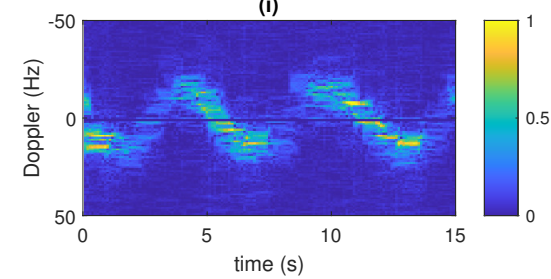

Fig. 10: Doppler spectra examples: sitting still in (a) sparse mode, (b) enhanced mode and (c) transmission status; body swing in (d) sparse mode, (e) enhanced mode and (f) transmission status; and walking in (g) sparse mode, (h) enhanced mode and (i) transmission status.

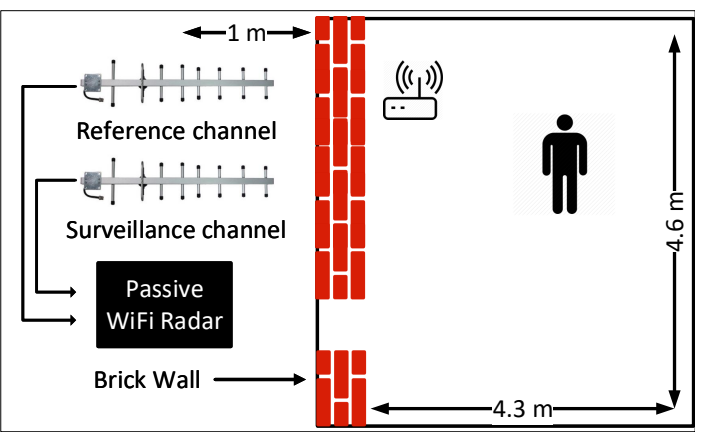

Fig. 11: Experiment layout.

\section{A. System Mode Comparison}

We first demonstrate our system's detection performance with different WiFi signal density, or duty cycles. We compare the Doppler signature during the WiFi idle status with both sparse and enhanced mode, and during the data transmission status. The transmission status is considered as the optimum signal for sensing (given the current settings) and this 'gold standard' is as the baseline reference when comparing to the other two modes in idle status.

Three activity classes are measured to demonstrate the feasibility our passive WiFi radar system. Each activity consist of different motion velocities, For example standing still encompasses a slow movement of the chest during breathing, body swing involves rocking on the spot with a medium velocity, and walking forward and backward within surveillance area typically consists of a wider range of faster motions. All obtained Doppler spectrograms are shown in Fig 10. As can be seen, sparse mode can give the correct Doppler pattern for breathing and body swing. The two peaks in Fig 10(a) in both the positive and negative domain represent the Doppler change during the process of inhalation and exhalation. Also the sinusoidal wave in Fig 10(d) shows the periodic characteristics associated with body swinging. Despite the fact that some noise appears in the spectrogram, the overall Doppler shape is distinguishable. As discussed before, the major limitation of the sparse mode is the maximum detectable velocity. This happens when a faster activity is performed, for example walking. Fig $10(\mathrm{~g})$ illustrates a Doppler signature that is difficult to interpret because of the walking speed goes beyond the Doppler boundary and appears in the other side of the Doppler domain, from the maximum positive Doppler bin to the negative domain or another way. In such a case, the sparse mode of passive WiFi radar can simply act as a motion detector.

Enhanced mode can be then activated for a better detection with the client simulator. Benefits of the higher frame rate, spectrogram in Fig 10(b), (e), (h) shows improved Doppler signatured when compare that in sparse mode. All three activities are clearly captured with distinguishable trace. However, there are some noise spread at different Doppler bin among the spectrogram. This is because of the time gap between the probe response frame that induces errors among correlation and FFT in CAF process.

As expected, transmission status deliveries the best performance in all scenarios. It outputs clear Doppler signature for all three activities and with lowest noise level in spectrogram. There are even some micro-Doppler can be seen across the walking activity in the Fig 10(i). But still, enhanced mode can 


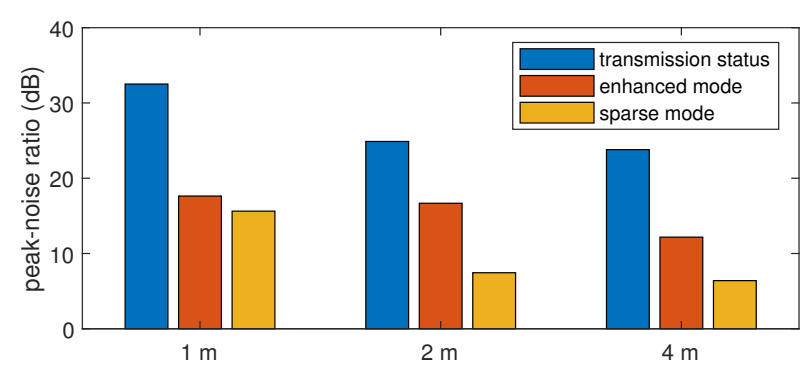

Fig. 12: Sensitivity of breathing detection at different distances.
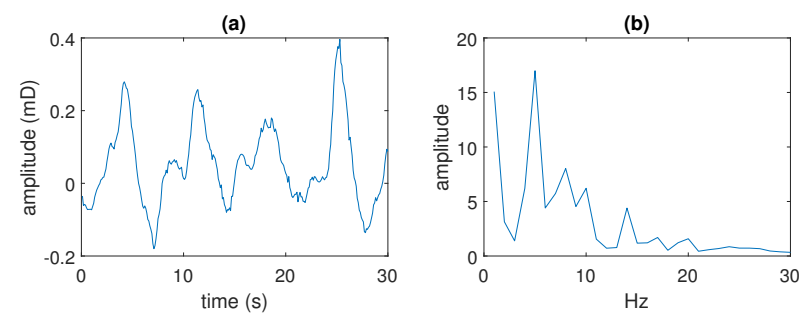

Fig. 13: (a) breathing signal from micro-Doppler and (b) breathing rate from FFT.

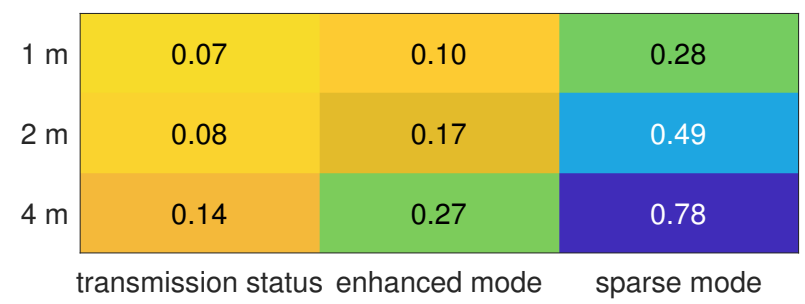

Fig. 14: Error rate of detected breathing rate versus distance.

provide close performance compare to that from transmission status.

\section{B. Breathing Detection}

To verify the sensitivity of the proposed system for breathing detection, a subject stood still and faced the surveillance antenna. Limited by the room size, the experiment was carried out at $1 \mathrm{~m}, 2 \mathrm{~m}$ and $4 \mathrm{~m}$ away from the wall under all three modes. We calculate the PNR of the Doppler peak which is caused by the chest movement and with the background noise in CAF surface. A higher PNR corresponds to an increased probability of detecting a breathing signal from the Doppler spectrogram, whereas a low PNR means more noise. The results are shown in Fig 12. As expected, the transmission status shows the best performance, following with the enhanced mode and sparse mode. The extracted breathing signal and rate suggests that breathing detection in transmission status is capable of covering the entire room, whereas enhanced mode and sparse mode can only cover part of the room.

We have shown the Doppler spectrogram for breathing detection in Fig 10(a), (b), (c). Here, Eq 9 was applied to the Doppler spectrogram to extract the breathing signal. Fig 13(a) shows an example of a 30 second breathing signal extracted
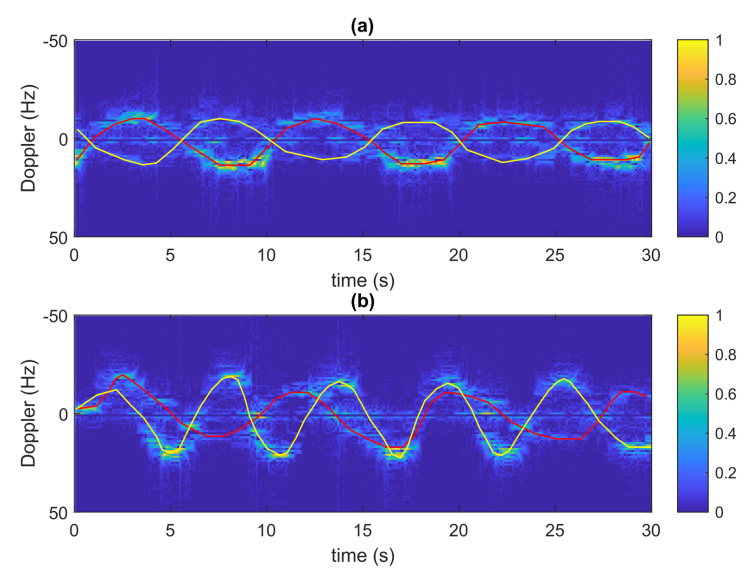

Fig. 15: Example of two people walking (a) same velocity, opposite direction, (b) different velocity, varying direction. The red and yellow lines are the Doppler trajectory for each person.

from Doppler spectrogram. The exhalation and inhalation phases of breathing can clearly be identified. Parameters such as the signal amplitude, frequency and periodicity in the breathing response can be potentially used for the identification of the subjects health condition [26]. A simple FFT was used to calculate the breathing rate, shown in Fig 13(b), which corresponding to the breathing signal in (a). We pick the maximum absolute value of the output as the frequency of the breathing rate, and avoid the first frequency component which may contain high noise. The dominant peak clearly indicates 4 repetitions (in 30 seconds) which equivalents to 8 breaths per minute.

Two minutes of breathing measurement were captured from each status, and the person was asked to breathe at a constant rate by counting the time. The rate of breathing rate was calculated over 30 seconds with a moving window of 1 second, which is expected to be constant for each measurement. We calculate the error rate for each scenario as shown in Fig 14. It can be seen that the best error rates are obtained with transmission status for all distances, whereas enhanced mode shows close error rates but slightly worse. Sparse mode has the worst performance and is unable to correctly detect the breathing rate beyond $2 \mathrm{~m}$.

\section{People counting}

Person detection experiments are typically based on data generated by a single person. However, the presence of multiple people is a more realistic in a real-world scenario. One such application scenario is the ability to count the number of people in locations like a shopping mall, train station or airport. Work [27] developed a WiFi CSI system to count a group of walking people, however this type system needs regularly calibration to cope the changing of surrounding environment. In comparison, our passive WiFi radar system does not require any calibration and provide meaningful Doppler signature. Enhanced mode was used in this experiment, since 


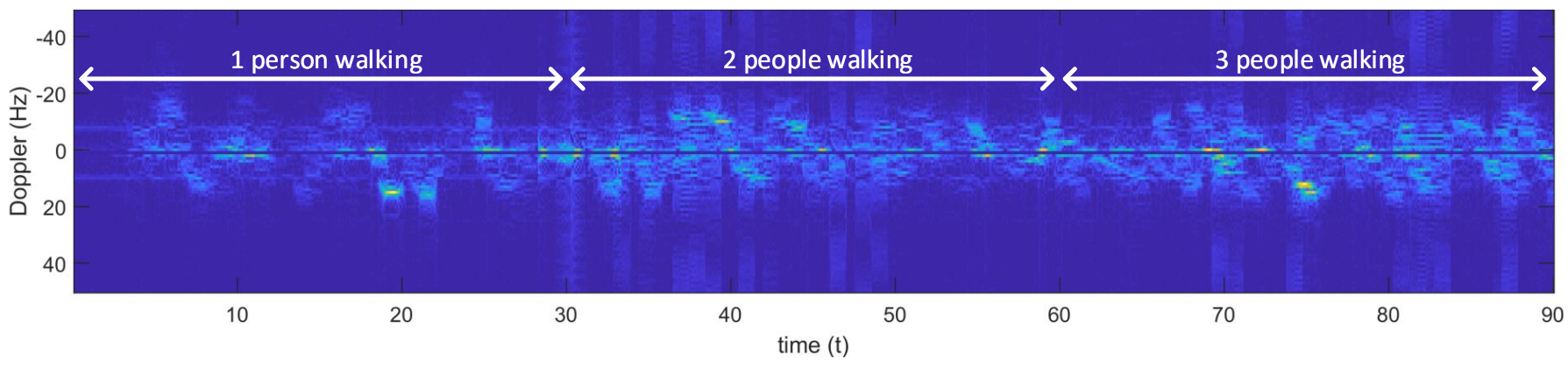

Fig. 16: Example of a random walk for (a) one person, (b) two people and (c) three people.

the sparse mode is not able to detect walking correctly as presented in Fig 10(g).

Fig 15(a) shows two people walking in same velocity but opposite direction in a straight line, e.g. one walk forward to the surveillance antenna while another one backward. The Doppler trajectory for each person is also labelled in different color. As it can be seen, there are two sinusoidal Doppler signature in the spectrogram, each represents a trajectory for one person. The Doppler signature for both person are clear with same periodicity and velocity, and almost identity direction. Fig 15 (b) shows two people walking in different velocity and remaining in a straight line. The spectrogram shows two sinusoidal Doppler signature but with different periodicity and velocity. These results show how the velocity of two people would affect spectrogram. Such Doppler spectrogram could potentially provide velocity for indoor tracking with proper tracking filter.

To simulate a more realistic scenario and demonstrate the difference in the number of people, maximum three people were asked to enter into the experiment room and walk in random direction. The Doppler spectrogram for random walking is shown in Fig 16. As it can be seen, the Doppler signature is much random and no longer a sinusoidal wave due to the direction is not in a straight line. Based on the velocity and duration, it can be easily distinguished from other body activities like a body swing. We can also see obvious difference in Doppler signature among the one person, two people and three people walking. One person shows a simple Doppler signature with a single trajectory, whereas two people have more complicated trajectory and three people show the most messy trajectory. This can be understood use the principle discussed in Fig 15 that the Doppler spectrogram is a combination of multiple Doppler signature from each moving person. By calculating the number of Doppler signature within certain period, it is possible to count the number of people. Such results could also be used for applications like people counting.

Fig 17 shows the PNR distribution in Doppler spectrogram as in Fig 16. These distributions clearly show the difference for different number of people walking. The one person case has the lowest PNR value, while three people walking has the highest PNR value. This is because of the multiple Doppler peaks which contain a higher PNR value than the single Doppler peak. To further verify the

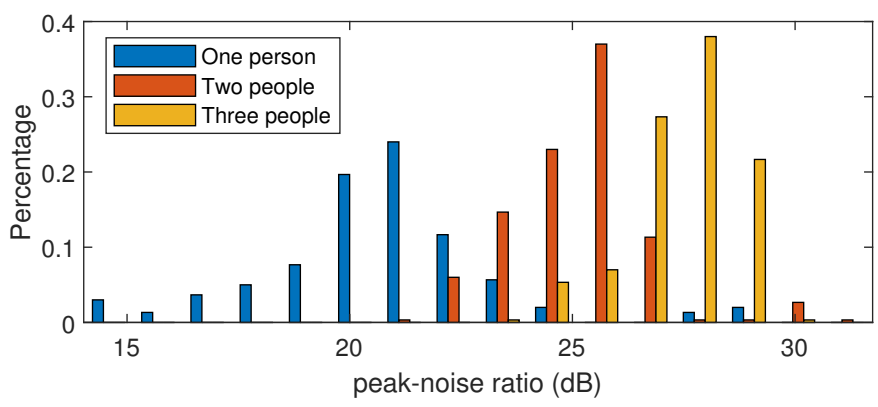

Fig. 17: Histogram of PNR distribution for one, two and three people.

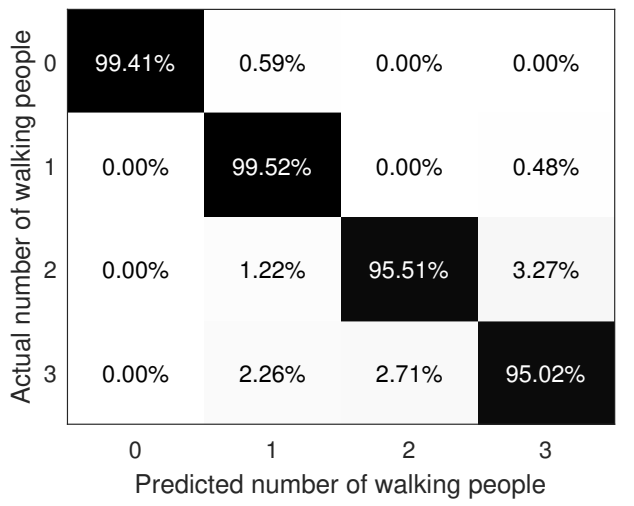

Fig. 18: Confusion matrix of people counting.

accuracy of people counting, a 10 minute measurements of one, two, and three people walking in a room were collected, including a control measurements for when the room was unoccupied. The resulting spectrograms were segmented into small samples consisting of a window size of half second. In total 5,051 samples were acquired, among them 4,206 were used as training samples and 845 were used as testing samples. A simple LeNet neural network [28] was used as the classifier. The classification results are shown in Fig 18, with an overall accuracy of $97.37 \%$. All classes reach more than $95 \%$ accuracy, especially the empty room and one person walking classes, which both are more than $99 \%$. The accuracy for multiple people walking slightly decreases to around $95 \%$ but remains at high level. Such performance illustrates the application potential for the proposed system to be used in occupancy detection and counting. 


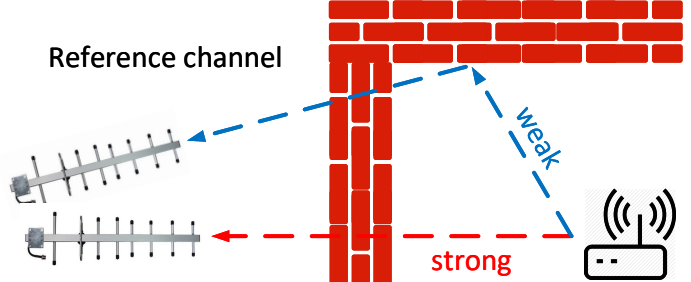

Fig. 19: Example of strong and weak reference channel.

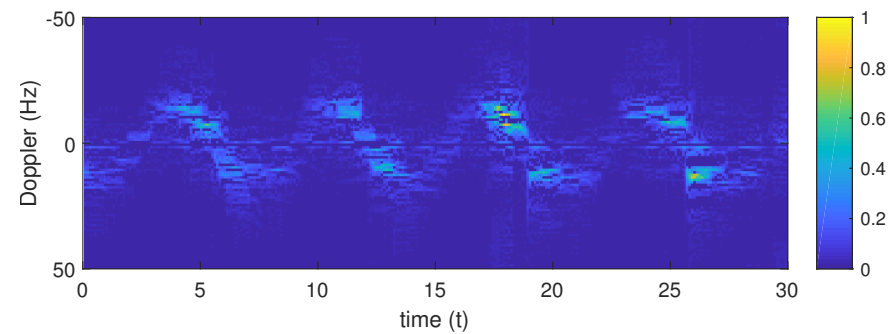

Fig. 20: The Doppler spectrogram of walking activity with weak reference channel.

\section{Weak Reference Channel}

One of the major challenges of our passive WiFi radar system is the stability of the reference channel. Consider in a real scenario, the reference channel may not always as strong as the experiment layout shown in Fig 11. When the antenna is not pointing to the WiFi AP directly, reference channel becomes weak that could bring additional noise the CAF surface. Thus, it is necessary to exam the detection performance under weak reference channel. A typical layout of strong and weak reference channel is illustrated in Fig 19. Recall the CAF processing in Eq 3, it assumes reference signal $S_{\text {ref }}$ perfectly recreate the transmitted signal from WiFi AP without time delays and Doppler shift. In terms of weak channel, additional time delay $\tau_{0}$ (from wall reflection) is added into the $S_{r e f}$. CAF surface with weak reference channel can be then rewritten as $C A F\left(\tau+\tau_{0}, f_{d}\right)$. Since $\tau_{0}$ does not affect the Doppler measurement, our passive WiFi radar system can still function in such layout. However, the received power is reduced due to the longer signal path and weaker reflections from wall. In this experiment, the reference antenna was placed to the WiFi AP with an angle of 45 degree. Here, we provide a Doppler spectrogram for walking as shown in Fig 20. Due to the weaker reference channel, Doppler signature is weaker than that in Fig 10(i) and Fig 15 , but it is still clear and distinguishable. This spectrogram shows that our passive WiFi radar can tolerate the imperfect reference channel and provide similar results.

\section{CONCLUSIONS}

In this paper, we have demonstrated a passive WiFi radar system which uses a stand-alone WiFi AP. This system does not require any specific modification to hardware nor firmware, and it can work with any commercial WiFi AP. Two modes are implemented to cope the sparse beacon frame during the WiFi idle status. The sparse mode uses a modified CAF to extract the beacon frame based on the power profile, while it only provides limited Doppler detection. The modified CAF shows an average of $8 \mathrm{~dB}$ improvement comparing to classical CAF below 100 frame rate. Enhanced mode takes advantage of external client simulator which increases the throughput of WiFi signal. Enhanced mode can deliver similar performance as compared to the case with full transmission status. We have demonstrated the potential of the proposed system in realworld deployment including the activity recognition (Section VI-A), TTW breathing detection (Section VI-B) and people counting (Section VI-C). It is envisioned that a stand-alone WiFi sensing system can surpass current WiFi system which requires either high frame rate or specific system layout.

Future works include the implementation of WiFi decoder to replace the energy curve for a better synchronization. Also, a comparison between our passive radar system and CSI system would be very helpful to further explore the potential of WiFi sensing.

\section{ACKNOWLEDGMENTS}

This work was funded under the OPERA Project, the UK Engineering and Physical Sciences Research Council (EPSRC), Grant EP/R018677/1.

\section{REFERENCES}

[1] C. J. Caspersen, K. E. Powell, and G. M. Christenson, "Physical activity, exercise, and physical fitness: definitions and distinctions for healthrelated research." Public health reports, vol. 100, no. 2, p. 126, 1985.

[2] M. Amjadi, K.-U. Kyung, I. Park, and M. Sitti, "Stretchable, skinmountable, and wearable strain sensors and their potential applications: a review," Advanced Functional Materials, vol. 26, no. 11, pp. 1678$1698,2016$.

[3] S.-R. Ke, H. Thuc, Y.-J. Lee, J.-N. Hwang, J.-H. Yoo, and K.-H. Choi, "A review on video-based human activity recognition," Computers, vol. 2, no. 2, pp. 88-131, 2013.

[4] A. Sciarrone, C. Fiandrino, I. Bisio, F. Lavagetto, D. Kliazovich, and P. Bouvry, "Smart probabilistic fingerprinting for indoor localization over fog computing platforms," in 2016 5th IEEE International Conference on Cloud Networking (Cloudnet). IEEE, 2016, pp. 39-44.

[5] H. Abdelnasser, M. Youssef, and K. A. Harras, "Wigest: A ubiquitous wifi-based gesture recognition system," in 2015 IEEE Conference on Computer Communications (INFOCOM). IEEE, 2015, pp. 1472-1480.

[6] Y. Wang, J. Liu, Y. Chen, M. Gruteser, J. Yang, and H. Liu, "Eeyes: device-free location-oriented activity identification using finegrained wifi signatures," in Proceedings of the 20th annual international conference on Mobile computing and networking. ACM, 2014, pp. 617-628.

[7] W. Wang, A. X. Liu, M. Shahzad, K. Ling, and S. Lu, "Devicefree human activity recognition using commercial wifi devices," IEEE Journal on Selected Areas in Communications, vol. 35, no. 5, pp. 11181131, 2017.

[8] W. Wang, A. X. Liu, and M. Shahzad, "Gait recognition using wifi signals," in Proceedings of the 2016 ACM International Joint Conference on Pervasive and Ubiquitous Computing. ACM, 2016, pp. 363-373.

[9] H. Wang, D. Zhang, J. Ma, Y. Wang, Y. Wang, D. Wu, T. Gu, and B. Xie, "Human respiration detection with commodity wifi devices: do user location and body orientation matter?" in Proceedings of the 2016 ACM International Joint Conference on Pervasive and Ubiquitous Computing. ACM, 2016, pp. 25-36.

[10] F. Colone, P. Falcone, C. Bongioanni, and P. Lombardo, "Wifi-based passive bistatic radar: Data processing schemes and experimental results," IEEE Transactions on Aerospace and Electronic Systems, vol. 48, no. 2, pp. 1061-1079, 2012.

[11] F. Colone, D. Pastina, P. Falcone, and P. Lombardo, "Wifi-based passive isar for high-resolution cross-range profiling of moving targets," IEEE Transactions on Geoscience and Remote Sensing, vol. 52, no. 6, pp. 3486-3501, 2013. 
[12] I. Milani, F. Colone, C. Bongioanni, and P. Lombardo, "Impact of beacon interval on the performance of wifi-based passive radar against human targets," in 2018 22nd International Microwave and Radar Conference (MIKON). IEEE, 2018, pp. 190-193.

[13] Q. Chen, B. Tan, K. Chetty, and K. Woodbridge, "Activity recognition based on micro-doppler signature with in-home wi-fi," in 2016 IEEE 18th International Conference on e-Health Networking, Applications and Services (Healthcom). IEEE, 2016, pp. 1-6.

[14] W. Li, B. Tan, R. J. Piechocki, and I. Craddock, "Opportunistic physical activity monitoring via passive wifi radar," in 2016 IEEE 18th International Conference on e-Health Networking, Applications and Services (Healthcom). IEEE, 2016, pp. 1-6.

[15] W. Li, B. Tan, and R. J. Piechocki, "Non-contact breathing detection using passive radar," in 2016 IEEE International Conference on Communications (ICC). IEEE, 2016, pp. 1-6.

[16] W. Li, B. Tan, Y. Xu, and R. J. Piechocki, "Log-likelihood clusteringenabled passive rf sensing for residential activity recognition," IEEE Sensors Journal, vol. 18, no. 13, pp. 5413-5421, 2018.

[17] K. Chetty, G. E. Smith, and K. Woodbridge, "Through-the-wall sensing of personnel using passive bistatic wifi radar at standoff distances," IEEE Transactions on Geoscience and Remote Sensing, vol. 50, no. 4, pp. 1218-1226, 2012.

[18] H. Wang, D. Zhang, Y. Wang, J. Ma, Y. Wang, and S. Li, "Rt-fall: A real-time and contactless fall detection system with commodity wifi devices," IEEE Transactions on Mobile Computing, vol. 16, no. 2, pp. 511-526, 2016.

[19] S. Tan and J. Yang, "Wifinger: leveraging commodity wifi for finegrained finger gesture recognition," in Proceedings of the 17th ACM international symposium on mobile ad hoc networking and computing. ACM, 2016, pp. 201-210.

[20] B. Tan, K. Woodbridge, and K. Chetty, "A real-time high resolution passive wifi doppler-radar and its applications," in 2014 International Radar Conference. IEEE, 2014, pp. 1-6.

[21] W. Li, B. Tan, and R. Piechocki, "Passive radar for opportunistic monitoring in e-health applications," IEEE journal of translational engineering in health and medicine, vol. 6, pp. 1-10, 2018.

[22] R. v. Nee and R. Prasad, OFDM for wireless multimedia communications. Artech House, Inc., 2000.

[23] S. M. Markalous, S. Tenbohlen, and K. Feser, "Detection and location of partial discharges in power transformers using acoustic and electromagnetic signals," IEEE Transactions on Dielectrics and Electrical Insulation, vol. 15, no. 6, pp. 1576-1583, 2008.

[24] $\mathrm{Ni}$ usrp $2921 . \quad$ [Online]. Available: http://sine.ni.com/nips/cds/view/p/lang/en/nid/212995

[25] Y. Ma, G. Zhou, and S. Wang, "Wifi sensing with channel state information: A survey," ACM Computing Surveys (CSUR), vol. 52, no. 3, p. 46, 2019.

[26] Y. S. Lee, P. N. Pathirana, C. L. Steinfort, and T. Caelli, "Monitoring and analysis of respiratory patterns using microwave doppler radar," IEEE journal of translational engineering in health and medicine, vol. 2, pp. $1-12,2014$.

[27] W. Xi, J. Zhao, X.-Y. Li, K. Zhao, S. Tang, X. Liu, and Z. Jiang, "Electronic frog eye: Counting crowd using wifi," in IEEE INFOCOM 2014-IEEE Conference on Computer Communications. IEEE, 2014, pp. 361-369.

[28] Y. LeCun et al., "Lenet-5, convolutional neural networks," URL: http://yann. lecun. com/exdb/lenet, vol. 20, p. 5, 2015. 